\title{
Características Morfológicas Métricas y No Métricas del Esternón del Individuo Mapuche
}

\author{
Metric and Non-metric Morphological Traits of the Sternum in Mapuche
}

\author{
Mariano del Sol ${ }^{* * * *}$; Bélgica Vásquez ${ }^{* * *}$ \& Mario Cantín ${ }^{* * * * * * *}$
}

\begin{abstract}
DEL SOL, M.; VÁSQUEZ, B. \& CANTÍN, M. Características morfológicas métricas y no métricas del esternón del individuo Mapuche. Int. J. Morphol., 32(1):351-356, 2014.

RESUMEN: El objetivo fue describir las características morfológicas métricas y no métricas del esternón de individuos Mapuche de sexo masculino y comparar estos parámetros con otras poblaciones del mundo. Se realizó un estudio descriptivo sobre 50 esternones secos de individuos Mapuche adultos de la región de la Araucanía, Chile, con edades comprendidas entre los 20 y 84 años. La longitud del esternón se midió en milímetros como la distancia combinada del manubrio y el cuerpo del esternón desde la incisura yugular a la unión xifoesternal, junto a la longitud del manubrio y cuerpo del esternón de manera independiente a lo largo del plano mediosagital. Para el proceso xifoides, se determinó también su longitud, ancho y grosor. Las características no métricas como forma y presencia de

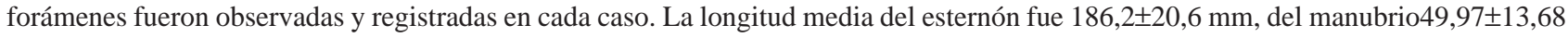

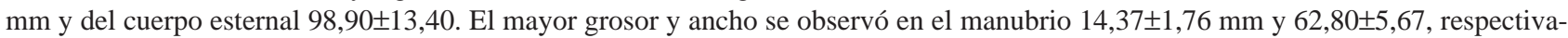
mente, seguido por el cuerpo esternal. El proceso xifoides mostró las menores dimensiones tanto en largo, ancho y grosor. Se observaron forámenes esternales (8\%) y xifoideos (12\%), y la forma bífida y de paleta fueron las más comunes en el proceso xifoides. La morfología del esternón permiten caracterizar rasgos antropológicos y forenses de tipo población-específica. Los rasgos métricos como la longitud combinada del esternón, podrían ser utilizados para discriminar sexo y estimar la estatura en el individuo Mapuche, mientras que los no métricos pueden ser relevantes para determinar variaciones biológicas, estimar edad o aspectos de interés clínico en la población Mapuche.
\end{abstract}

PALABRAS CLAVE: Esternón; Osteometría, Mapuche; Rasgos métricos, Rasgos no métricos; Características morfológicas.

\section{INTRODUCCIÓN}

De acuerdo al XVII Censo Nacional de Población (2002), en Chile existe 4,6\% de población indígena, de los cuales el individuo Mapuche representa el 87,3\%, distribuidos principalmente en regiones al sur de Chile. Aunque existen más de 600.000 Mapuches en Chile, las investigaciones de antropología física realizadas en esta población son escasas en relación a los estudios de antropología social y cultural. Los estudios describen diámetros e índices cefálicos, curvas craneales, diámetros e índices faciales, índices claviculares, evaluación de la curvatura cervical, evaluación postural, entre otros aspectos (del Sol \& Olave, 1996; Henriquez et al., 2003; del Sol \& Hunter, 2004; del Sol, 2005, 2006). Respecto a estudios antropológicos realizados en esternón de individuos Mapuche, son aún más escasos (del Sol et al., 1990).
Al igual que varios restos óseos, el esternón presenta características morfológicas métricas y no métricas poblacionales específicas, lo que le ha dado una considerable atención en los estudios forenses y antropológicos.

La determinación del sexo, estatura y edad de los restos esqueléticos está bien estudiado y ampliamente documentado. Bajo este contexto, estudios realizados en diversas poblaciones del mundo, tales como la India, Europa, África, Canadá y América del Norte, han demostrado que el análisis del esternón puede conducir a una determinación precisa del sexo basándose en características morfológicas, tales como, longitud del cuerpo, longitud combinada del manubrio y cuerpo, ancho de la incisura esternal, ancho del esternón a nivel de la cuarta costilla, entre otras (Dahiphale

\footnotetext{
Facultad de Medicina, Universidad de La Frontera, Temuco, Chile.

** Centro de Investigación en Ciencias Biomédicas, Universidad Autónoma de Chile, Temuco, Chile.

*** Universidad de Tarapacá, Arica, Chile.

***** CIMA, Facultad de Odontología, Universidad de la Frontera, Temuco, Chile.
} 
et al., 2002; Atal et al., 2008; Ramadan et al., 2010; Bongiovanni \& Spradley, 2012; Singh et al., 2012, 2013; Singh \& Pathak, 2013a).

Por su parte, la estimación de la estatura a partir de restos óseos humanos constituye uno de los pasos básicos en la práctica arqueológica y forense. En el contexto arqueológico, estudios han demostrado que la estatura adulta se puede considerar como un indicador sintético que refleja las condiciones de nutrición y salud bajo las que se desarrolla una población (Bogin, 1999). Desde el punto de vista forense, el establecimiento de la estatura es un factor muy significante en la identidad de los restos óseos humanos. Frecuentemente, el análisis de los huesos largos permite estimar de manera muy precisa la estatura (De Mendonça, 2000; Hasegawa et al., 2009; Ilayperuma et al., 2010). Sin embargo, los huesos largos pueden no estar presentes en todos los casos, lo que exige el posible uso de otros elementos esqueléticos disponibles tales como el esternón. Desde esta perspectiva, Menezes et al. (2009) utilizando ecuaciones de regresión lineal, han establecido la estatura de individuos adultos del sur de la India analizando la longitud del esternón.

La edad de los individuos también puede ser estimada a través de rasgos no métricos mediante el análisis del grado de fusión (completo, parcial o ausente) de los diferentes elementos del esternón, tales como, las articulaciones manubrio-esternal y xifo-esternal (Gautam et al., 2003; Kaneriya et al., 2013). El objetivo fue describir las características morfológicas métricas y no métricas del esternón de individuos Mapuche de sexo masculino y comparar estos parámetros en otras poblaciones del mundo.

\section{MATERIAL Y MÉTODO}

Se realizó un estudio descriptivo sobre 50 esternones de individuos Mapuches, adultos de sexo masculino, con edades comprendidas entre los 20 y 84 años, en la Facultad de Medicina de la Universidad de La Frontera, Chile. Los huesos se prepararon a través de maceración suave para eliminar el tejido blando y secados para realizar las mediciones.

Se excluyeron los esternones con cualquier tipo de fractura visible, patología, reparaciones quirúrgicas o deformidad/anormalidad, para mantener una buena integridad estructural de la muestra y poder determinar las características métricas y no métricas. En el estudio osteométrico se realizaron mediciones lineales con la posición anatómica del esternón. Se determinó la longitud individual del manubrio, del cuerpo del esternón (mesoesternón) y la longitud total del esternón identificada como la combinación entre manubrio y mesoesternón entre la incisura yugular a la articulación mesoxifoídea. Todas las longitudes se determinaron en el plano mediosagital. También se determinaron el ancho y grosor del manubrio y mesoesternón. Para el proceso xifoides, se determinó su longitud, ancho y grosor.

Los resultados fueron tabulados para determinar el valor medio y desviación estándar de las mediciones. Además, estos valores fueron comparados con los reportados previamente en estudios osteológicos en otras poblaciones.

\section{RESULTADOS}

Entre las características métricas estudiadas, la longitud total del esternón presentó una media de 186,2 $\pm 20,6 \mathrm{~mm}$. El 52\% de los esternones tuvo un rango de longitud entre los 170 y $199,9 \mathrm{~mm}$, seguido por un $22 \%$ con medidas entre 150 164,9 mm y el $22 \%$ midió $200 \mathrm{~mm}$ o más. Sólo dos piezas esternales se ubicaron en el rango de 164,9-169,9 mm.

El cuerpo del esternón presentó la mayor longitud con una media de $98,90 \pm 13,40$, mientras que para el manubrio fue de 49,97 $\pm 13,68 \mathrm{~mm}$. El mayor grosor y ancho se observó en el manubrio $14,37 \pm 1,76 \mathrm{~mm}$ y $62,80 \pm 5,67$, respectivamente, seguido por el cuerpo esternal, el cual en la mayoría de los casos fue más grueso en el tercio superior. El proceso xifoides mostró las menores dimensiones tanto en largo, ancho y grosor (Tabla I).

Las características no métricas más interesantes son las diferentes formas observadas en el proceso xifoides: bífido en 21 casos (42\%), ovalado (10\%), de paleta (38\%), otras (10\%). Además, se observó la presencia de forámenes esternal y xifoideo. En cuatro casos (8\%) fue encontrado un foramen esternal, localizado en el tercio inferior del cuerpo, el cual presentaba sus diámetros vertical y transversal más o menos iguales. Por otra parte, un foramen xifoideo se observó en 6 casos (12\%), 3 casos con un foramen único y otros 3 dobles. En los casos de foramen único fue de forma redondeada y tamaño variable.

Respecto a los promedios de los rasgos métricos actuales del esternón en hombres de diferentes grupos poblacionales de estudios osteológicos, la literatura señala que la mayor longitud esternal se presentó en población de Croacia $(208,6 \pm 1,46)$, seguido de la población africana, europea y de USA (Selthofer et al., 2006; Macaluso, 2010; Bongiovanni \& Spradley). La población India mostró los valores más bajos en diferentes estudios. Los detalles en relación a las mediciones del manubrio y cuerpo esternal se muestran en la Tabla II. 
Tabla I. Dimensiones (Media_DE) de los rasgos métricos del esternón de sujetos Mapuche de sexo masculino.

\begin{tabular}{lcccccc}
\hline Dimensiones & \multicolumn{2}{c}{ Manubrio } & \multicolumn{2}{c}{ Cuerpo } & \multicolumn{2}{c}{ Proceso xifoides } \\
$(\mathbf{m m})$ & Media & DE & Media & DE & Media & DE \\
\cline { 2 - 7 } Largo & 49,97 & 13,68 & 98,90 & 13,40 & 37,96 & 10,00 \\
Ancho máximo & 62,80 & 5,67 & 38,04 & 5,22 & 21,90 & 6,03 \\
Grosor máximo & 14,37 & 1,76 & 13,40 & 2,30 & 7,20 & 1,71 \\
\hline
\end{tabular}

Tabla II. Valores promedio de rasgos métricos del esternón pertenecientes a sujetos de sexo masculino en diferentes grupos poblacionales. Las mediciones fueron realizadas sobre material osteológico.

\begin{tabular}{|c|c|c|c|c|c|c|c|c|}
\hline \multirow[t]{2}{*}{ Estudios } & \multirow[t]{2}{*}{ Población } & \multirow{2}{*}{$\begin{array}{c}\text { Esternón } \\
\begin{array}{c}\text { Largo } \\
(\mathbf{m m})\end{array}\end{array}$} & \multicolumn{3}{|c|}{ Manubrio } & \multicolumn{3}{|c|}{ Cuerpo } \\
\hline & & & $\begin{array}{l}\text { Largo } \\
(\mathbf{m m})\end{array}$ & $\begin{array}{l}\text { Ancho } \\
(\mathbf{m m})\end{array}$ & $\begin{array}{c}\text { Grosor } \\
(\mathbf{m m})\end{array}$ & $\begin{array}{l}\text { Largo } \\
(\mathbf{m m})\end{array}$ & $\begin{array}{l}\text { Ancho } \\
(\mathbf{m m})\end{array}$ & $\begin{array}{c}\text { Grosor } \\
(\mathbf{m m})\end{array}$ \\
\hline Ashley (1956) & Europa & 156,9 & 52,2 & --- & --- & 104,7 & 33,7 & --- \\
\hline & África & 142,6 & 45,9 & --- & --- & 96,5 & 30,9 & --- \\
\hline Dahiphale (2002) & India & $\begin{array}{c}142,196 \\
( \pm 11,297)\end{array}$ & $\begin{array}{c}48,458 \\
( \pm 5,596)\end{array}$ & --- & --- & $\begin{array}{c}94,427 \\
( \pm 9,521)\end{array}$ & --- & --- \\
\hline $\begin{array}{l}\text { Gautam et al. } \\
\text { (2003) }\end{array}$ & India & 149,00 & 53,00 & --- & --- & 95,00 & --- & --- \\
\hline $\begin{array}{l}\text { Torwalt \& Hoppa } \\
\text { (2005) }\end{array}$ & Canadá & 142,34 & 54,43 & --- & --- & 109,41 & --- & --- \\
\hline $\begin{array}{l}\text { Sel thofer } e t a l \text {. } \\
\text { (2006) }\end{array}$ & Croacia & $\begin{array}{c}208,6 \\
( \pm 1,46)\end{array}$ & $\begin{array}{c}5,52 \\
( \pm 0,36)\end{array}$ & $\begin{array}{c}68,2 \\
( \pm 8,0)\end{array}$ & $\begin{array}{c}1,26 \\
( \pm 0,19)\end{array}$ & $\begin{array}{l}10,97 \\
( \pm 1,44)\end{array}$ & $\begin{array}{c}3,07 \\
( \pm 0,53)\end{array}$ & $\begin{array}{c}1,00 \\
( \pm 0,11)\end{array}$ \\
\hline $\begin{array}{l}\text { Hunnargi et al. } \\
\text { (2008) }\end{array}$ & India & 117,25 & 51,99 & --- & --- & 89,17 & --- & --- \\
\hline Macaluso (2010) & $\begin{array}{l}\text { África del Sur } \\
\text { (Negros) }\end{array}$ & $\begin{array}{l}174,24 \\
( \pm 11,5)\end{array}$ & $\begin{array}{c}48,51 \\
( \pm 4,94)\end{array}$ & $\begin{array}{c}52,08 \\
( \pm 4,76)\end{array}$ & --- & $\begin{array}{c}98,74 \\
( \pm 9,54)\end{array}$ & $\begin{array}{c}31,77 \\
( \pm 5,10)\end{array}$ & --- \\
\hline $\begin{array}{l}\text { Menezes } \text { et al. } \\
\text { (2011) }\end{array}$ & India (Sur) & $\begin{array}{c}141,2 \\
( \pm 10,7)\end{array}$ & --- & --- & --- & --- & --- & --- \\
\hline $\begin{array}{l}\text { Bongiovanni \& } \\
\text { Spradley (2012) }\end{array}$ & USA & $\begin{array}{c}154,97 \\
( \pm 10,85)\end{array}$ & $\begin{array}{c}51,84 \\
( \pm 4,93)\end{array}$ & --- & $\begin{array}{l}104,80 \\
( \pm 9,8)\end{array}$ & --- & --- & --- \\
\hline $\begin{array}{l}\text { Puttabanthi et al. } \\
\text { (2012) }\end{array}$ & India & $\begin{array}{c}139,55 \\
( \pm 21,093)\end{array}$ & $\begin{array}{c}47,48 \\
( \pm 12,029)\end{array}$ & $\begin{array}{c}53,32 \\
( \pm 13,497)\end{array}$ & --- & $\begin{array}{c}92,36 \\
( \pm 14,032)\end{array}$ & $\begin{array}{c}33,41 \\
( \pm 6,72)\end{array}$ & --- \\
\hline $\begin{array}{l}\text { Singh et al. } \\
\text { (2012) }\end{array}$ & India & --- & --- & $\begin{array}{c}54,91 \\
( \pm 5,44)\end{array}$ & --- & --- & $\begin{array}{c}33,53 \\
( \pm 5,49)\end{array}$ & --- \\
\hline $\begin{array}{l}\text { Kaneriya et al. } \\
\text { (2013) }\end{array}$ & India (Surat) & $\begin{array}{l}149 \\
( \pm 4) \\
\end{array}$ & $\begin{array}{c}52 \\
( \pm 8) \\
\end{array}$ & --- & --- & $\begin{array}{c}98 \\
( \pm 18) \\
\end{array}$ & $\begin{array}{c}39 \\
( \pm 9) \\
\end{array}$ & --- \\
\hline
\end{tabular}

\section{DISCUSIÓN}

El esternón presentan características antropométricas particulares con gran valor antropológico y forense para poblaciones específicas como la Mapuche, pero también estas características son de utilidad para clínicos y cirujanos, ya que este hueso es un estructura crítica en la pared anterior del torax, el cual puede verse afectado por traumas, procedimientos quirúrgicos, diversas anomalías anatómicas y procesos patológicos, algunos de los cuales requieren tratamiento (Restrepo et al., 2009).

Entre las características métricas estudiadas, la longitud total del esternón presentó una media de 186,2 $\pm 20,6$ $\mathrm{mm}$, con un rango entre 150 y $200 \mathrm{~mm}$. Estos resultados concuerdan con la mayoría de las investigaciones realiza- das sobre piezas esternales óseas, con rangos similares, aunque el promedio fue mayor para la población Mapuche en la mayoría de los casos (Tabla II). En indios, Gautam et al. reportaron una longitud combinada de manubrio y cuerpo del esternón que va desde $101 \mathrm{~mm}$ a $192 \mathrm{~mm}$, con una media de 149,00 mm para hombres, similar a los promedios reportados por Jit \& Bakshi (1986) y Dwight (1890) (147,08 $\mathrm{mm}$ y $164,1 \mathrm{~mm}$, respectivamente). Sólo la población croata presentó una longitud superior a la nuestra, con un promedio de longitud de 208,6 $\pm 1,46 \mathrm{~mm}$ (Selthofer et al.).

En relación a la determinación del sexo basándose en características morfológicas del esternón, Gautam et al. en un estudio realizado en 56 esternones de hombres indios, 
indicaron que si la longitud combinada de un esternón excede los $161 \mathrm{~mm}$, el individuo es de sexo masculino. Nuestros resultados se correlacionan positivamente con lo observado por los autores, destacando además que el número de esternones utilizado en ambos estudios fueron similares. De manera similar Ashley (1956) formuló la "regla 149", según la cual un esternón masculino debe superar los $149 \mathrm{~mm}$ de longitud. Así, la longitud combinada del esternón sería un rasgo métrico adecuado para discriminar positivamente a individuos de sexo masculino en población Mapuche.

Por otra parte, los esternones de individuos mapuches presentaron características no métricas de interés, tales como, forámenes esternales y xifoideos. Se ha sugerido la posibilidad de utilizar tales rasgos como caracteres antropológicos; además, las características morfológicas que pueden ser observadas y descritas, pero que no se pueden medir, tienen probablemente mayor significado desde el punto de vista antropológico que las medidas de diámetro e índice (Singh \& Pathak, 2013b). Variantes no métricas han sido utilizadas en antropología física para determinar variaciones biológicas entre poblaciones, diversidad genética y racial, sexo, edad, así como características de estrés por actividades u ocupaciones. Desde esta perspectiva, sería de gran utilidad ampliar el conocimiento de estos posibles marcadores étnicos para caracterizar de manera más precisa la población Mapuche desde la visión antropológica y forense.

La presencia de un foramen esternal (redondo u oval), puede corresponder a una anomalía congénita o del desarrollo óseo común, debido probablemente al retraso del desarrollo, fusión incompleta o defectuosa de los centros de osificación de un par de esternebras del mesoesternon o xiphiesternon (Rodríguez-Vásquez et al., 2013). La presencia de un foramen es típicamente único, y raramente múltiple (McCormick et al., 1998). La muestra de población Mapuche estudiada presentó una incidencia del 8\%, la cual no es comparable con otras poblaciones reportadas en la literatura, ya que duplica a la población Inglesa $(3,8 \%)$, Europea $(4,0 \%)$ e India $(4,1 \%)$ (Paterson, 1904; Ashley; Jit \& Bakshi), y es inferior a poblaciones del Noroeste de India $(11,9 \%)$, Africana $(13,3 \%)$ y de Papua $(15,4 \%)$ (Singh \& Pathak, 2013b; Schultz, 1944; Ashley; Steive \& Hintzsche, 1925). Así, las características no métricas del esternón de individuos Mapuche, podrían se utilizadas como marcador antropológico; sin embargo, se debe considerar que serían necesarios muestreos de mayor representatividad. Resulta relevante esta información específica para hombres de población Mapuche, ya que la presencia de un foramen esternal es de cuidado en sujetos que reciben acupuntura o la aspiración de la médula ósea del esternón debido al riesgo de complicaciones asociadas (por ejemplo, hemorragia y taponamiento cardíaco) (Restrepo et al.).
Por otra parte, la evaluación de la diversidad morfológica del proceso xifoides para la realización del diagnóstico y el tratamiento quirúrgico de las enfermedades relacionadas con este hueso ha tenido un mayor interés clínico estos últimos años. En nuestra muestra, el proceso xifoides presentó una longitud promedio de 37,96 $\pm 10,00 \mathrm{~mm}$,

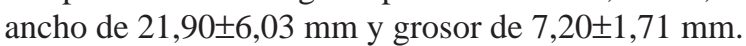

Respecto a la forma observada en los procesos xifoides, del Sol \& Olave (1998) describieron previamente las características morfológicas del proceso xifoides en Mapuche, observando que las formas bífidas y en paleta son las más frecuentes. Concordamos con los reportes de Singh \& Pathak (2013b), en el cual el proceso xifoide de la población de noreste de la India, tiene un alto grado de variabilidad en su longitud, forma (bífida, en espada, triangular, rectangular u oval) y tamaño (pequeño, largo y estrecho). La forma variable del proceso xifoides, como romboide a triangular u oval, fue descrita por primera vez por Goodman et al. (1983) utilizando imágenes de TC, quienes también encontraron que el proceso xifoides en su extremo distal a menudo tenía una calcificación irregular grumoso o de apariencia bífida. Akin et al. (2011) estudiaron a 577 pacientes y observaron que el extremo distal del proceso xifoides presentaba tres tipos: única $(62,6 \%)$, doble $(32,8 \%)$ o triple $(4,6 \%)$. El foramen xifoideo estuvo presente en el $12 \%$ de nuestros casos, y sólo se observó de manera única o doble, mientras que Akin et al. también han reportado la existencia de tres y cuatro forámenes. De esta manera, tener un acabado conocimiento de la morfología de esta parte del esternón puede ser útil para comprender y diagnosticar la enfermedad del proceso xifoides, particularmente en procedimientos de cirugía o inyección.

Concluimos que la morfología del esternón permiten caracterizar rasgos antropológicos y forenses de tipo población-específica. La longitud individual y combinada del esternón son los rasgos métricos más útiles para discriminar entre sexos masculino y femenino, y muestran una buena correlación para estimar la estatura (Malacuso \& Lucena, 2014), mientras que los rasgos no métricos son relevantes para determinar variaciones biológicas, estimar edad o aspectos de interés clínico en la población Mapuche.

DEL SOL, M.; VÁSQUEZ, B. \& CANTÍN, M. Metric and nonmetric morphological traits of the sternum in Mapuche. Int. J. Morphol., 32(1):351-356, 2014.

SUMMARY: The aim was to describe the metric and nonmetric morphological traits of the sternum in Mapuche males and to compare these parameters with other world populations. A descriptive study on 50 dry adult Mapuche male individuals Sterna from the region of Araucania, Chile, aged between 20 and 84 
years were reviewed. The length of the sternum was measured in $\mathrm{mm}$ as the combined length of the manubrium and body of the sternum from the incisura jugularis to the mesoxiphoid junction, and manubrium and body of the sternum independently along the midesagittal plane. To the xiphoid process, was also determined length, width and thickness. The non-metric features such as shape and presence of foramina were observed and recorded in each case. In the sternum, mean length was $186.2 \pm 20.6 \mathrm{~mm}$, in the manubrium $49.97 \pm 13.68 \mathrm{~mm}$ and for the body of the sternum was $98.90 \pm 13.40$. The greater thickness and width was observed on the manubrium $14.37 \pm 1.76$ and $62.80 \pm 5.67 \mathrm{~mm}$, respectively, followed by the body of the sternum. The xiphoid process showed the smaller both in length, width and thickness. Sternal and xiphoid foramina was observed in $8 \%$ and $12 \%$, and bifid and paddle shape were the most common observed in the xiphoid process. The morphology of the sternum allows forensic anthropological and characterize features in a specific population. Metric traits as the combined length of the sternum, could be used to discriminate sex and estimate the height in the individual Mapuche, while nonmetric may be relevant to determine biological variation, estimating age or aspects of clinical interest in the Mapuche population.

KEY WORDS: Sternum; Osteometry, Mapuche; Metric traits, non-metric traits; Morphological characteristics.

\section{REFERENCIAS BIBLIOGRÁFICAS}

Akin, K.; Kosehan, D.; Topcu, A. \& Koktener, A. Anatomic evaluation of the xiphoid process with 64-row multidetector computed tomography. Skeletal Radiol., 40(4):447-52, 2011.

Ashley, G. T. The human sternum. The influence of sex and age on its measurement. J. Forensic Med., 3(1):27-43, 1956.

Atal, D. K.; Murari, A. \& Naik, S. K. Role of Sternal Index in Determination of Gender. Indian J. Forensic Med. Pathol., 3:34, 2008.

Bogin, B. Patterns of Human Growth. Cambridge, Cambridge University Press, 1999.

Bongiovanni, R. \& Spradley, M. K. Estimating sex of the human skeleton based on metrics of the sternum. Forensic Sci. Int., 219(1-3):290.e1-7, 2012.

Dahiphale, V. P.; Baheete, B. H. \& Kamkhedkar, S. G. Sexing the human sternum in Marathwada region. J. Anat. Soc. India, 51(2):162-7, 2002.

Del Sol, M.; Vieira, M. C. \& Olave, E. Estudo morfométrico do esterno no grupo étnico Mapuche. Arq. Anat. Antropol., 41:2116, 1990 .

Del Sol, M. \& Olave, E. Índices claviculares en el grupo étnico mapuche. Rev. Chil. Anat., 14(2):183-8, 1996.
Del Sol, M. \& Olave, E. El proceso xifoides del esternón mapuche. Rev. Chil. Anat., 16(1):101-6, 1998.

Del Sol, M. \& Hunter, K. Evaluación postural de individuos mapuche de la zona costera de la IX región de Chile. Int. J. Morphol., 22(4):339-42, 2004.

Del Sol, M. Índice cefálico en un grupo de individuos Mapuches de la IX Región de Chile. Int. J. Morphol., 23(3):241-6, 2005 .

Del Sol, M. Índices faciales en individuos mapuche. Int. J. Morphol., 24(4):587-90, 2006.

De Mendonça, M. C. Estimation of height from the length of long bones in a Portuguese adult population. Am. J. Phys. Anthropol., 112(1):39-48, 2000.

Dwight, T. Sternum as an Index of Sex, Height, and Age. $J$. Anat. Physiol., 24(Pt. 4):527-35, 1890.

Gautam, R. S.; Shah, G. V.; Jadav, H. R. \& Gohil, B. J. The human sternum as an index of age \& sex. J. Anat. Soc. India, 52(1):20-3, 2003.

Goodman, L. R.; Teplick, S. K. \& Kay, H. Computed tomography of the normal sternum. AJR Am. J. Roentgenol., 141(2):219-23, 1983.

Hasegawa, I.; Uenishi, K.; Fukunaga, T.; Kimura, R, \& Osawa. M. Stature estimation formulae from radiographically determined limb bone length in a modern Japanese population. Leg. Med. (Tokyo), 11(6):260-6, 2009.

Henríquez, J.; Fuentes, R.; Sandoval, P. \& Muñoz, A. Análisis de la estabilidad ortostática cráneocervical en adultos jóvenes mapuches. Int. J. Morphol., 21(2):149-53, 2003.

Hunnargi, S. A. ; Menezes, R. G.; Kanchan, T.; Lobo, S. W.; Binu, V. S.; Uysal, S.; Kumar, H. R.; Baral, P.; Herekar, N. G. \& Garg, R. K. Sexual dimorphism of the human sternum in a Maharashtrian population of India: a morphometric analysis. Leg. Med. (Tokyo), 10(1):6-10, 2008.

Ilayperuma, I.; Nanayakkara, G. \& Palahepitiya, N. A model for the estimation of personal stature from the length of forearm. Int. J. Morphol., 28(4):1081-6, 2010.

Jit, I. \& Bakshi, V. Incidence of foramina in North Indian sterna. J. Anat. Soc. India, 33:77-84, 1984.

Kaneriya, D.; Umarvanshi, B.; Patil, D.; Mehta, C.; Chauhan, K. \& Vora, R. Age determination from fusion of the sternal elements. Int. J. Basic Applied Med. Sci., 3(2):22-9, 2013.

Macaluso, P. J. Jr. The efficacy of sternal measurements for sex estimation in South African blacks. Forensic Sci. Int., 202(13):111.e1-7, 2010. 
Macaluso, P. J. Jr. \& Lucena, J. Stature estimation from radiographic sternum length in a contemporary Spanish population. Int. J. Legal Med., 2014. (Epub ahead of print)

McCormick, W. F.; Flournoy, L. E.; Rogers, N. L. \& Ross, A. H. An unusual case of multiple mesosternal foramina. $J$. Forensic Sci., 43(3):706-7, 1998.

Menezes, R. G.; Kanchan, T.; Kumar, G. P.; Rao. P. P.; Lobo, S. W; Uysa, S.; Krishan, K.; Kalthur, S. G.; Nagesh, K. R. \& Shettigar, S. Stature estimation from the length of the sternum in South Indian males: a preliminary study. $J$. Forensic Leg. Med., 16(8):441-3, 2009.

Paterson, A. M. The Human Sternum. London, William and Norgate, 1904. pp.42-67.

Puttabanthi, S.; Velichety, S. D.; Padi, T. R.; Boddeti, R. K. \& Priyanka, J. R. Sexing of unknown adult human sterna by metrical analysis. Int. J. Biol. Med. Res., 3(2):1516-9, 2012.

Ramadan, S. U.; Türkmen. N.; Dolgun, N. A.; Gökharman, D.; Menezes, R. G.; Kacar, M. \& Kos,ar, U. Sex determination from measurements of the sternum and fourth rib using multislice computed tomography of the chest. Forensic Sci. Int., 197(1-3):120.e1-5, 2010.

Restrepo, C. S.; Martinez, S.; Lemos, D. F.; Washington, L.; McAdams, H. P.; Vargas, D.; Lemos, J. A.; Carrillo, J. A. \& Diethelm, L. Imaging appearances of the sternum and sternoclavicular joints. Radiographics, 29(3):839-59, 2009.

Rodríguez-Vázquez, J. F.; Verdugo-López, S.; Garrido, J. M.; Murakami, G. \& Kim, J. H. Morphogenesis of the manubrium of sternum in human embryos: a new concept. Anat. Rec. (Hoboken), 296(2):279-89, 2013.

Schultz, A. H. Age changes and variability in gibbons: a morphological study on a population sample of a man-like ape. Am. J. Phys. Anthropol., 2:1-129, 1944.

Selthofer, R.; Nikolic', V.; Mrcela, T.; Radic'́, R.; Leksan, I.; Rudez, I. \& Selthofer, K. Morphometric analysis of the sternum. Coll. Antropol., 30(1):43-7, 2006.

Singh, J.; Pathak, R. K. \& Singh, D. Morphometric sex determination from various sternal widths of Northwest Indian sternums collected from autopsy cadavers: A comparison of sexing methods. Egyptian J. Forensic Sci., 2:18-28, 2012.

Singh, J. \& Pathak, R. K. Morphometric sexual dimorphism of human sternum in a north Indian autopsy sample: sexing efficacy of different statistical techniques and a comparison with other sexing methods. Forensic Sci Int., 228(13):174.e1-10, 2013a.
Singh, J. \& Pathak, R. K. Sex and age related non-metric variation of the human sternum in a Northwest Indian postmortem sample: a pilot study. Forensic Sci. Int., 228(13):181.e1-12, 2013b.

Stieve, H. \& Hintzsche, E. Ucher die form des menschlichen, Brustbeins. Z. Morphol. Anthropol., 23:361-410, 1925.

Torwalt, C. R. \& Hoppa, R. D. A test of sex determination from measurements of chest radiographs. J. Forensic Sci., 50(4):785-90, 2005.

Dirección para Correspondencia:

Prof. Dr. Mariano del Sol

Facultad de Medicina

Universidad de La Frontera

Temuco

CHILE

Email: mariano.delsol@ufrontera.cl

Recibido : 17-10-2013

Aceptado: 22-01-2014 